\title{
Project therapeutic singular of a patient with severe dementia: seeking greater proximity to the ESF
}

\begin{abstract}
Currently, we aim humanization of health and comprehensive care to users. To that end, rethought action strategies and production of care, such as the Therapeutic Project Singular - PTS. The study aimed to strengthen the knowledge of PTS in multiprofessionality, draw up a PTS to assist the staff of the Family Health Strategy in the care of the selected patient and his family, emphasizing the importance of adopting this strategy as a routine health services. home visits were carried out to know the life history of the patient and the history of present illness, were made complete history and physical examination, applied to Rabbit Range held the Mini-Mental State Examination (MMSE) and designed genogram and eco-map, elucidating patient relations with the environment and with other family members. Eighteen goals have been put in place to better care and preventing new illnesses/comorbidities. The PTS allowed to know the reality of the family in relation to the conditions of the patient and the "hidden" needs to the FHS team and even neglected by the family, as well as allowed the strengthening of ties with the ESF, verifying that the study expanded the vision of team regarding the family, from which it is possible to work the curative action and introducing preventive actions. It was also possible to see how much is fundamental greater attention to hear a patient or his family, trying to be attentive to detail and make guidelines that will change the reality of a family and ameliorate problems experienced by facilitating access to the defendants health needs.
\end{abstract}

Volume 5 Issue 4 - 2018
Perez JP, Carli Roque J, Tereza Lais Menegucci

Department of Nursing and Medicine Courses, University of Marília, Brazil

Correspondence: Tereza Lais Menegucci Zutin, Department of Nursing and Medicine Courses, University of Marília, Brazil, Email laiszutin@hotmail.com

Received: June 18,2018 | Published: July 17, 2018

\section{Introduction}

Traditionally, the model of care used with health user not considered as an active subject of their treatment, did not involve his family and did not value their history, culture, everyday life and quality of life. The main focus of attention was the disease. This model has undergone changes since the creation of the Unified Health System (SUS), adding value characteristics of knowledge and opinions of users/families in the construction of the treatment plan. ${ }^{1}$

The humanization of health and comprehensive care to SUS users are goals that have been almejadas by workers and public health professionals today. To that end, rethought action and care production strategies that put the user at the center of attention and your health as an end, like the Singular Therapeutic Project (PTS). ${ }^{2}$ The PTS is a set of articulated therapeutic approaches proposed for an individual or collective subject, the result of collective discussion of an interdisciplinary team, matrix support, if necessary. Generally, it is dedicated to more complex situations. In fact, it is a discussion of the variation of "clinical case". 3

The PTS incorporates interdisciplinary notion that collects the contribution of various specialties and different professions. So after a shared assessment of the user conditions are agreed procedures in charge of several members of the multidisciplinary team, called the reference team. Thus, the reference teams undertake the construction of individual responsibility and stable link between health team and user/family. Each professional reference shall be responsible for accompanying people throughout the treatment that organization, providing the intervention of other professionals or support services consonant necessary and ultimately ensuring high and continuous follow-up on another instance of the system. ${ }^{1}$ From this perspective, the present work aims to strengthen the ownership of the knowledge of PTS in the context of multiprofessionality, draw up a PTS to assist the team at the Nations Park ESF in the care of MTR patients, their caregivers and their families, emphasizing the importance of adopting this strategy as practice to be incorporated into routine health services in the NHS, by managers, health professionals, such as academics and researchers, from a theoretical reflection of the subject, since it is known that the PTS represents a time of all staff involved and all opinions are important to help understand the individual and/or family with some complex health needs as a whole. Because it is an account of teaching and learning experience, there was no need for his referral to the Research Ethics Committee in accordance with Resolution No. 196/1996 of the National Health Council.

\section{Method}

This is an experience report, drawn from the experience of an academic undergraduate course in medicine during the first half of 2017. In order to integrate the practical activities of the discipline of Primary Attention to Health, was presented to proposal to draw up a PTS of a user and family ESF where practical activities occurred. The choice of PTS emerged through the case of the suggestion by the community health workers during a staff meeting, where it was mentioned the condition of the patient and the need for a closer and strengthening of ties with the ESF. home visits to perform the PTS 17, 18, 19, 24 and 25 April 2017. From the analysis of this information, we list the problems and seek the literature data of psychopathology, medications, current treatments and other questions demanded by the 
user. On this information, we prepared beside her goals we wanted to achieve, as well as the care plan. In the last meeting, we present you the PTS, to be validated from its analysis and make the considerations deemed pertinent.

\section{Case report}

Subject search: Date of Birth: 18/10/1949 - 66 years; Women; White color; Birth: Marilia/SP; remote origin: Parana and Mato Grosso; Current origin: Marilia (35 years); Education: Illiterate; Marital status: Married. History of present illness: There are approximately four years, MTR, which until then lived normally and took care of your life and your home, started a forgetting frame places that used to store everyday objects and also has to keep the objects in wrong places (examples: pot inside the wardrobes, shoe inside the refrigerator etc). After a few days started to perform repetitive questions and short time (minutes) already forgot what was asked and what was obtained in response. In one month, patient developed symptoms similar to the current and caregiver reports that realizes gradual worsening daily. Today, the patient does not adequately address the questions posed to her, does not recognize his daughter, does not know her husband's name, does not know age and has totally disoriented in time and space. Personal history: Patient disoriented in time and space, with a total loss of the memory and disjointed conversations, rambles perfectly. Carrier Type II diabetes mellitus for about 10 years, about 8 years ago hypertension, Alzheimer for approximately 4 years and hypothyroidism (time did not mention disease). Does not have drug and food allergies, he held a surgery 28 years ago ( + cesarean tubal ligation). 7 has hospitalizations and 6 for obstetric causes (4 PVEs, 2 abortions and cesarean 1). 1 month ago 8 days was admitted to $\mathrm{HCII}$ because uncontrolled hypertension and DM. Makes use of geriatric diaper sleeping, it has power tending to pasty held orally, having good acceptance, but eats compulsively and has perversion of appetite (eat food remains, inedible objects, earth, flowers, weeds etc). He is currently in the care of her daughter due to the advanced stage of Alzheimer's disease.

living habits: Patient lives daily in the daughter of the house, where it receives the necessary care (medications, food and bath). Is outpatient treatment for Alzheimer's neurologist. Have good night's sleep without interruption from $21 \mathrm{~h}$ to $6 \mathrm{~h}$, in the afternoons, sleeps about 20 minutes every day. It has never been alcoholic and or smoke, receives power as one of the family.

Household and socioeconomic conditions:MTR live in brick houses with paved street. The house is rented (monthly amount of R $\$ 550.00$ ) and has 5 rooms ( 2 bedrooms, living room, kitchen and bathroom), sanitation and garbage collection. The main caregiver of MTR is a daughter of 43 years old and live with 4 people (son in law, 45; son, 28 years; grandchildren, 24 years and 22 years). MTR is not retired and receives no sickness, but the caretaker states that have started retirement process with an attorney and is awaiting the final decision.

\section{Results and discussion}

After the choice of the user, we started the first stage of PTS: the host and the construction of the genogram and eco-map (Figure 1), through home visits, whose objectives were explained to the family, allowing us to know its dynamics, clarifying questions of their members in relation to the monitoring to be performed. The ecomap provides an enlarged view of the family, by designing the supporting structure and depicting the link between the family and the world. This tool connects the circumstances to the environment and shows the link between family members and community resources, consisting of a diagram of the relationship between the family and the community that helps to assess the support and supports available and their use by the family. ${ }^{4}$ Data were obtained through interviews with the daughter (caregiver) MTR. This required the establishment of a bond and trust in an attempt to provide a comfortable situation to report the particulars of his story. The display of the genogram and eco-map allows us to know the family of internal and external structures. The interview took place in the current residence of $\mathrm{M}$, which is currently in the care of her daughter due to the advanced stage of Alzheimer's disease. Lady $\mathrm{M}$ was unable to provide information due to disorientation and amnesia caused by the disease; the other members of the house did not attend the interview by any of the visits are on site. During the interview, $\mathrm{N}$ us reported that the patient $\mathrm{M}$ is living in your home in order to receive the necessary care (medications, food and bath) and $\mathrm{M}$ has 4 more children in addition to the daughter and the weekend is always in the house of some children, making a rotation between them so that every weekend she can stay at home a different child. In the same house that $\mathrm{M}$ lies, home to more five people, including his daughter and caregiver (43 years); son in law, 45; son, 28 years; grandchildren, a 24 year old and one 22 years. The daughter reported that the $\mathrm{M}$ now has a conflicting relationship with her husband after 10 years of marriage because he became alcoholic, aggressive and possessed extramarital relationships. Besides the visit to the home of the children on weekends, the only occasions where $\mathrm{M}$ is leaves his home to go to the ESF and to go to the Clinic Medical Specialties, which makes treatment for Alzheimer's neurologist. aggressive and possessed extramarital relationships. Besides the visit to the home of the children on weekends, the only occasions where $\mathrm{M}$ is leaves his home to go to the ESF and to go to the Clinic Medical Specialties, which makes treatment for Alzheimer's neurologist. aggressive and possessed extramarital relationships. Besides the visit to the home of the children on weekends, the only occasions where $\mathrm{M}$ is leaves his home to go to the ESF and to go to the Clinic Medical Specialties, which makes treatment for Alzheimer's neurologist.

Because of the lack of sanity M, could not establish the current bonds of relationships with other family members. However, the caretaker informed us that all always had strong bonds with M, except for her husband, as mentioned above. Thus, the genogram and ecomap might contribute to the acquisition of information about the family, showing the need to invest in care for caregivers, avoiding their illness, given that the patient $\mathrm{M}$, despite his good physical condition, has no sanity mental and requires intensive care. It is concluded that these instruments can be adopted by the ESF team as a way to approach the family, so this feels participant information collection process as well as possible to know the family regarding the family structure, culture, life cycle, and inter-relationships. ${ }^{4}$

\section{Mini mental state examination (MMSE)}

The Mini-Mental State Examination (MMSE), a brief mental status examination or Folstein test is a short questionnaire of 30 points used to track back cognitive impairments (Figure 2). It is commonly used inmedicineto trackinsanity. It is also used to estimate the severity of cognitive impairment at a specific time and follow the course of cognitive changes in an individual over time, thus making it an effective means of documenting the response of the individual to treatment. In about 10 minutes including measuring functionsarithmetic, memory, and Guidance. ${ }^{5}$ 


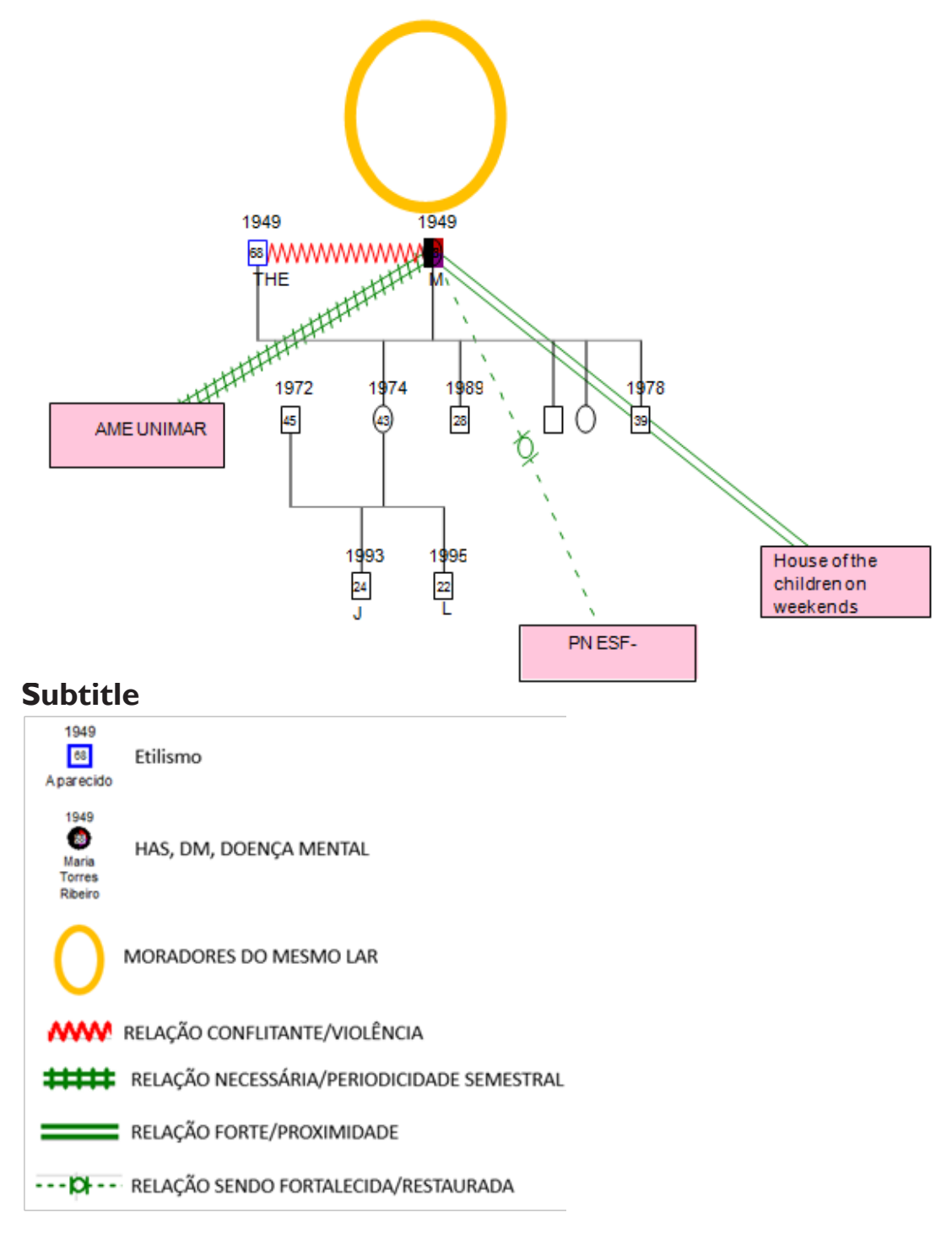

Figure I Genogram and ecomap.

The MMSE is composed of two parts: one covering orientation, memory and attention (maximum points $=21$ ) and the other which deals with specific skills such as naming and comprise (maximum points $=9$ ), amounting to a total score of 30 points. ${ }^{5}$ Higher values of score indicate greater cognitive performance. The survey addresses issues of recent memory and record of immediate memory, temporal and spatial orientation, attention and calculation and language aphasia, apraxia and constructional ability. Any score less than 27 (out of 30) is effectively normal (intact). Below that, the score may indicate severe cognitive impairment ( $\leq 9$ points), moderate $(10-20$ points) or mild (21-24 points) Gross score may need to be corrected according to education and age. low or very low scores are strongly correlated withinsanityAlthough other mental disorders can also lead to abnormal results in the MMSE test. The presence of a purely physical problems may also interfere with the interpretation is not properly considered; for example, a patient may not be able to hear or read instructions properly or may have a motor deficit that affects the ability to write or draw.
The patient $\mathrm{M}$ is the result was 04 points, indicating a severe cognitive impairment, dementia signal. In items "guidance", "attention and calculation" and "Evocation" the patient had a zero score and items "immediate memory" and "Language", the patient had a low score, which indicates a rapid progression above and even with the medication was not possible to avoid this prognosis, indicating failure in clinical treatment and depletion of measures that can improve the quality of life of the patient.

\section{Dementia}

Dementia is defined as the presence of multiple cognitive deficits manifested sufficiently severe so as to compromise the quality of life of affected individuals and their family members. ${ }^{6,7}$ Its diagnosis is based on the presence of memory decline and other higher cortical functions, for example, of language (aphasia), the ability to perform coordinated movements (apraxia), to recognize and identify objects, abstraction (agnosia), organization and the ability of sequencing and planning. ${ }^{8}$ Alzheimer's disease is recognized as the main cause of the 
dementia syndrome, considered one of the most painful conditions of adult life. ${ }^{9}$ The first visible signs of this disease, as well as other pathological processes in the elderly, is usually the occurrence of a functional disturbance that impacts negatively on daily activity, this signal being often overlooked. ${ }^{10}$ To identify possible cases are combined psychometric instruments that seek to measure, quantitatively, the individual cognition levels and clinical assessment itself by the doctor. ${ }^{8.11}$ In this context, it is of great importance that the affected patients have their diagnosis performed in the shortest possible time, enabling monitoring properly, One of the most important goals for the clinical management of affected patients is the recovery or maintenance of quality of life ${ }^{10}{ }^{\text {since the limitations that }}$ this condition imposes affect the freedom to move and the self-esteem of the diagnosed patient. ${ }^{2}$ Besides the loss in quality of life affected by dementia patient, the required care and time are particularly critical for patients in advanced stages of the disease. ${ }^{12,13}$

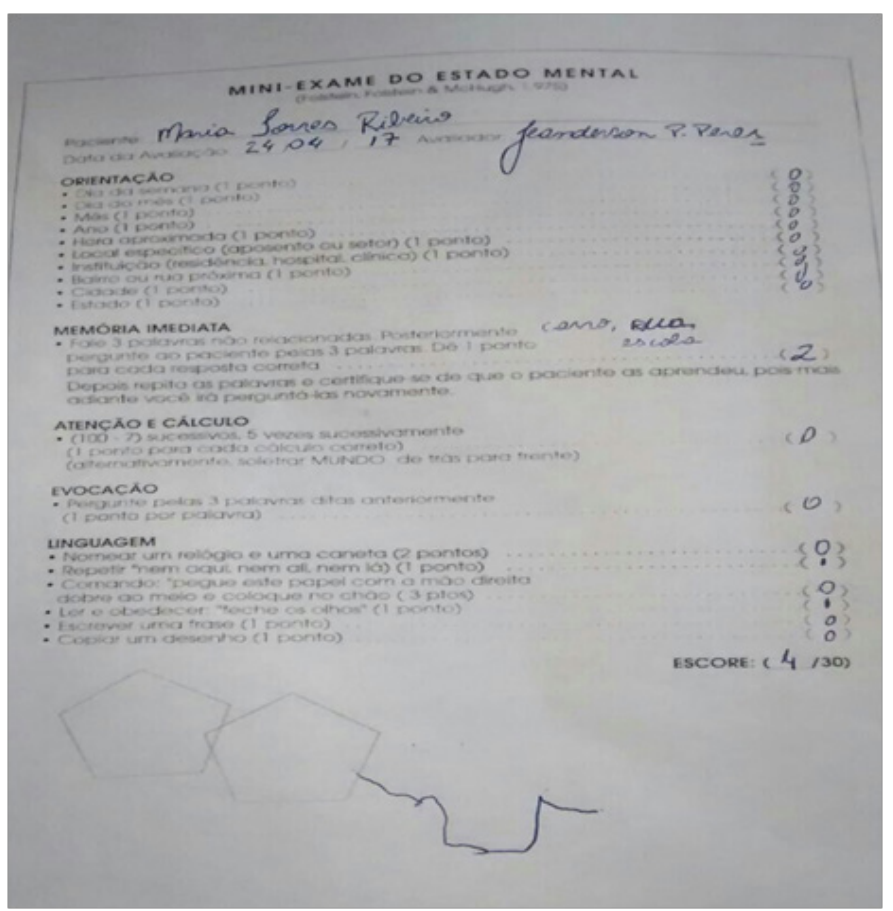

From:Assessing Cognitive components

Figure 2 Mini-examination MTR patient's mental status.

Family caregivers, who often are the pillars of support for these patients suffer from great emotional, psychological and economic. ${ }^{9}$ Also, dementia is one of the health problems that most grow in importance and number of cases, ${ }^{8}$ a fact that is due to the increase of the population in older age groups, due to the demographic transition experienced throughout the world. A recent and significant event in the context of dementia resulting from the fifth edition of the Diagnostic and Statistical Manual of Mental Disorders (DSM-5), published in 2013 is the change in some known aspects of the disease. Regarding the nomenclature used, the DSM-5 no longer uses the term "dementia" for believing that this has become stigmatized and poorly accepted among affected patients. In its place came to be used to naming "neurocognitive disorders", differentiating between light and greater disorder. In addition, it was recognized that the memory was not the first area to be affected by all types of dementia and has become required an evaluation of the cognitive performance of the patient compared to others in terms of z-scores, as it evaluates the difference between the results compared to the average in terms of standard deviations.

\section{Rabbit scale}

The introduction of the Family Health Program was an attempt to reorganize primary care in the country, which established the Home Visit, as a differential instrument performance of the GP. It is expected that an efficient and problem-solving strategy to be able to solve $80 \%$ of the demand of the health of a community services, as this demand is concentrated in a few problems, making it the centerpiece in the process of reorganization of networks and systems of health. Health professionals, in turn, must have the technical capacity to identify and prioritize social needs in health, organize knowledge and instruments in technological models of intervention setting and assess the impact of interventions. Besides the technical ability, should add bargaining power for intersectoral practice and ability to provide scientifically sound evidence to support the regulatory task of the modern state in the health field.

Within this problematic, it becomes evident the need for a tool that allows prioritize home visits as an essential action within the work process of the inserted multi-professional teams in USF. The ESF uses the registration form families, called A plug, which makes up the chart of the family, which allows the healthcare team to know the living conditions of people in their area, and is the initial source of contact and data collection relatives. Contains data on the number of people who make up the family, age, sex, literacy profiles, occupation, school children, whether they are studying or out of school, diseases or health conditions, and conditions housing, sanitation, use of health services, leisure and transport. It is characterized as a source of information on morbidity, points to a collective analysis by proposing that health teams make consolidated these areas chips for identifying the morbidity in the constitution of their families, For this purpose and to establish priorities in the visit, a family risk scale based on the record of the Primary Care Information System (SIAB) was prepared. This scale is based on sentinels of risk are assessed by the first home visit health agent (CHA). The results underscore the resident relationship/room as an important indicator of risk assessment as well as the applicability of the scale as a prioritization tool both home visits as the team's investment. The "Rabbit scale" emerges, then, as an assessment tool and monitoring of social and economic reality in the context of life of each family, recognizing the real health needs in the context of ESF.

The authors suggest that only the systematic use of rabbit scale as demand reorganization instrument, and subsequent evaluation of its impact on the community, you can confirm its applicability in the family physician's role. ${ }^{14}$

Applying the scale rabbit family studied (Table 1), we find:

Mental deficiency: 3 points

Unemployment: 2 points

Chronic disease: 2 points

Illiteracy: 1 point

resident relations /room $>1: 3$ points

Thus, we obtain a total score $=11$ points and found that this family fits the risk rating: R3, ie a family requires assistance because of the many presented vulnerabilities. This evaluation allowed to see the need for the proposed goals for better family care, framing actions that 
could assist the family in regard to socioeconomic actions also because we know that the quality of socio-economic life is a determinant of health and interferes directly in the healthcare process -disease. So when we get monthly diapers to the patient $\mathrm{M}$, among other actions as specified in the immediate goals and short-term, we can see that it was of fundamental importance to the family as the caregiver said that with this monthly spending less.

Table I Rabbit Scale and Savassi

\begin{tabular}{|c|c|c|}
\hline Data from A the A & & Score \\
\hline Bedridden & & 3 \\
\hline Denctencla Physics & & 3 \\
\hline Mental retardation & & 3 \\
\hline Fermentation conditions saneamento & & 3 \\
\hline Desnortric (serious) & & 3 \\
\hline drug addict & & 2 \\
\hline unemployment & & 2 \\
\hline Analfabensmo & & I \\
\hline Less than six months & & I \\
\hline Greater than seventy years & & $\mathrm{I}$ \\
\hline Hipertension arterial stamina & & I \\
\hline \multirow[t]{3}{*}{ Relocation: } & It is valued that I & 3 \\
\hline & Equals & 2 \\
\hline & It is $17|I| 0 \mid$. that I & 0 \\
\hline Total score & Risk rating & \\
\hline Score 5 or 6 & $\mathrm{RI}$ & \\
\hline Score 7 or 8 & $\mathrm{R} 2$ & \\
\hline Score 8 to 9 & R3 & \\
\hline
\end{tabular}

From: Family Risk Scale Application as prioritization tool of home visitation. ${ }^{15}$

\section{Goals and responsibilities division}

\section{Immediate and short-term goals}

i. Making home visits weekly to verify control of blood glucose and blood pressure (function intended for ACS, academic, nursing staff) - held goal.

ii. patient relationship strengthening and caring with the ESF (function intended for ACS and academic) - held goal.

iii. Discuss the case with the NASF and refer patient to nutritional assessment and guidance as to the type of diet needed to control hypertension and DM (function intended for academic and nutritionist NASF) held goal.

iv. Request requests for laboratory tests, checking: Complete blood count, lipid profile, fasting blood glucose, glycated hemoglobin, sodium, potassium, urea, creatinine, TSH, free T4, urine I and feces Parasitological (function intended for academic and medical) - Goal fulfilled.

v. Mark appointment for MTR for directions and optimization of drugs - held goal.

vi. Take the patient to the ESF for oral assessment and prevention of oral cancer - held goal.

vii. Check the need for medicine against intestinal parasites (function intended for medical and academic) - meta performed. viii. Inform the caregiver that many ongoing drug MTR are available free on the ESF and it should go to the unit to remove them instead of buying them (function intended for academic) - held goal.

ix. Diapers supply is available in the unit (function intended for academic and pharmacy staff) - held goal.

x. Fill request form to receive monthly adult diapers (function intended for academic and pharmacy staff) - held goal.

xi. Visit MTR spouse (chronic alcoholic) and try to establish link with the ESF (function intended for academic and ACS) - held goal, but not successful because the user does not receive the professional and did not want treatment or approach the ESF.

xii. Orientation relative to the aid Continuous Cash Benefit (BPC) of the Organic Law of Social Assistance - LOAS (function intended for academic) - held goal.

xiii. Guide caregivers on the importance of vaccination against influenza and ask to take M to the ESF to receive the same (function intended for academic) - held goal.

xiv. Guide caregiver referring to the right that has to receive medication for Alzheimer free (high-cost process), and instruct her to talk to the neurologist responsible for the patient to provide the request of the medication (function intended for academic) - meta held

\section{Medium-term targets}

i. Observe the resolution of appetite perversion that the patient has. If after the treatment of intestinal parasite persists frame, evaluating whether to initiate specific drug treatment (intended to function Medical).

ii. Monthly monitoring to assess caregiver burden and can help the caregiver, avoiding their illness (function intended for academic and ACS).

iii. Home medical consultation schedule for the husband of $\mathrm{M}$ (function intended for academic and medical) - held goal, but not successful because the user does not receive the professional and did not want treatment or approach the ESF.

iv. Discuss the case with the NASF and forward husband patient for psychological evaluation and guidance about the dangers of alcohol abuse for personal and family health (function intended for academic and NASF the psychologist) - goal accomplished, but not successful because the user does not receive professional and did not want treatment or approach the ESF.

\section{Conclusion}

The development of this work allowed the academic and staff of the Family Health Strategy better meet the family of the reality studied in relation to $\mathrm{M}$ pathologies as well as meet needs "hidden" to the FHS team and even neglected by the family, as well as allowed and a closer family bond strength to the FHS. Thus, it is concluded that this type of study is extremely important as it extends the vision of the team in relation to the family reality, highlighting the critical issues and potential vulnerabilities, from which you can work the curative action, and Equally important, introducing preventive measures seeking to prevent further illnesses and seek a behavioral remodeling. 
It was also possible to achieve the 14 goals listed in the short term, see how much greater attention from healthcare professionals is vital to listen to a patient or his family, trying to be attentive to detail and make guidelines that can change reality a family as well as alleviate problems experienced by facilitating access to the defendants health needs. As an academic, my time is short and the ESF will not be able to continue the listed targets the medium term, but stress the importance of continuing the same both for the next academic (as) here will as by the ESF team.

\section{Acknowledgments}

None.

\section{Conflict of interest}

The author delcears that there is no conflict of interest.

\section{References}

1. Pinto MD, Jorge MSB, Pinto AGA. Singular therapeutic projects in the production of comprehensive care: a collective construction. Rev Text Context Enferm. 2011;20(3):493-502.

2. Silva EP, Melo FABP, Sousa MM. unique therapeutic project as a practical strategy multiprofessionality in health care. Journal of Health Sciences. 2013;17(2):197-202.

3. Brazil. Ministry of Health. Department of Health Care. Technical Center for National Policy of Humanization. extended clinic, referral team and unique therapeutic project. 2nd ed. Series B. Basics Texts of Health Brasília; 2007.

4. Mello DF, Vieira CS, Simpionato, et al. Genogram and ecomap: Possible uses in health strategy família.VerBrasCresDesenv Hum. 2005;15(1):79-89.
5. Folstein MF, Folstein SE, Mchugh PR. "Mini-mentalstate". The practical method is grading the cognitive state of patients is theclinician. JournalofPsychiatricResearch. 1975;12(3):189-198.

6. APA. DiagnosticandStatistical Manual of Mental Disorders, 4th ed. Washington DC: American Psychiatric Association; 2000.

7. Fagundes SD, Silva MT Thees Mfrs, Pereira MG. PrevalenceofdementiaamongelderlyBrazilians: a systematicreview. Sao Paulo Med J. 2011;129(1):46-50.

8. Almeida OP. Mini-Mental State Examination and the diagnosis of dementia in Brazil. ArqNeuropsiquiatr. 1998;56(3B):605-612.

9. Ferri CP, Prince M, Brayne C, et al. Global prevalenceofdementia: a Delphi consensus study. Lancet. 2005;366(9503):2112-2117.

10. Lorenzo RA, Veras RP. Mini-Mental State Examination: psychometric characteristics in elderly outpatients. Journal of Public Health. 2006;40(4):712-719.

11. Brucki SMD, Nitrini R, Caramelli P PHF, et al. Suggestions for utilizationofte mini mentalstateexamination in Brazil. Arquivos de Neuro-Psiquiatria. 2003;61(3B):777-781.

12. Schultz-K Larsen, Lomholt RK. Kreiner S. Mini-Mental Status Examination: a short form the MMSE was as accurate to the original MMSE in predictingdementia. J ClinEpidemiol. 2007;60(3):260-267.

13. Siberski J. Dementiaand DSM-5: Changes, costandconfusion. AgingWell. 2012;5(6):12-16.

14. Assessing Cognitive components. 2013.

15. Rabbit Flg, Savassi Lcm. Family Risk Scale application prioritization as a tool for home visitation. Journal of Medicine of Family and Community. 2004;1(2):19-26. 